\title{
Mitochondrial Dysfunction and Resuscitation in Sepsis
}

\author{
Albert J. Ruggieri, Richard J. Levy, MD, and Clifford S. Deutschman, MS, MD, FCCM \\ Dept of Anesthesiology and Critical Care (AJR, CSD), University of Pennsylvania School of \\ Medicine, Philadelphia PA 19104-4283, the Division of Anesthesiology and Pain Medicine (RJL), \\ Children's National Medical Center, Washington DC 20010 and the Stavropoulos Sepsis \\ Research Program (AJR, RJL, CSD), University of Pennsylvania School of Medicine, \\ Philadelphia PA 19104-4283
}

\section{Introduction}

Sepsis is among the most common causes of death in intensive care unit patients in both North America and Europe. In the US it accounts for upwards of 250,000 deaths each year [1]. Despite this, the importance of sepsis as a public health problem is under-appreciated. This in-part stems from changing definitions. The dictionary definition invokes the presence of dividing micro-organisms in the blood. However, this has proven to be too narrow. The first systematic approach to the classification of sepsis, the 1992 Society of Critical Care Medicine (SCCM)/American College of Chest Physicians (ACCP) Consensus Conference, clarified some of the diagnostic criteria and also first introduced the concept of the systemic inflammatory response syndrome (SIRS) [2]. This entity was defined on the basis of temperature, heart rate, respiratory rate and white blood cell counts. While useful in defining entry criteria for clinical studies, this approach has proven to be too general and nonspecific. Therefore, the 2001 revision jointly sponsored by the SCCM and the European Society of Intensive Care Medicine (ESICM) is based on the presence of general variables, inflammatory variables, hemodynamics, organ dysfunction and tissue perfusion.

Significantly, the concept that sepsis of necessity involves infection has been eliminated. This is important for two reasons. First, no organism is ever identified in upwards of 50\% of patients who die with a diagnosis of sepsis. Second, systemic infection and similar inflammatory states give rise to a more recently appreciated entity, the multiple organ dysfunction syndrome (MODS) [3]. This clinically-defined common final pathway may develop in patients with severe infection but also following severe trauma, aortic aneurysm rupture, amniotic fluid embolism and a host of other conditions. The development of MODS suggests that the pathogenesis of sepsis cannot be due solely to invading organisms but rather must reflect the host response to some severe insult. The search for a primary defect and the mechanism by which it impairs function in a broad array of cells and organs has been the focus of intense investigation for several decades.

\footnotetext{
(C) 2010 Elsevier Inc. All rights reserved.

Correspondence to Dr Deutschman at Dulles 781A/HUP, 3400 Spruce Street, Philadelphia PS 19104-4283, (215) 662-3758, deutschcl@uphs.upenn.edu.
}

Publisher's Disclaimer: This is a PDF file of an unedited manuscript that has been accepted for publication. As a service to our customers we are providing this early version of the manuscript. The manuscript will undergo copyediting, typesetting, and review of the resulting proof before it is published in its final citable form. Please note that during the production process errors may be discovered which could affect the content, and all legal disclaimers that apply to the journal pertain. 


\section{Mitochondrial Function and Energy Production}

Investigations into the pathobiology of sepsis have most recently focused on cellular and sub-cellular processes that are common to most cells and organs. One possibility would be a defect in the production of energy. This translates to an abnormality in the production of adenosine triphosphate (ATP) and therefore in the function of mitochondria.

Mitochondria are cellular organelles characterized by a unique double membrane structure that maintain intracellular homeostasis via a number of key functions. Most important of these is the production of energy that can then be consumed elsewhere in the cell. In this process, ATP is produced from adenosine diphosphate (ADP) and consumed. A description of normal mitochondrial function is required before studies examining mitochondrial failure can be entertained as a cause of septic pathophysiology and the development of MODS.

The initiation of mitochondrial energy production in fact does not occur in mitochondria but most often takes place in the cytoplasm. This process, glycolysis (Fig 1), is a series of reactions by which glucose (derived most often from food but also synthesized by organs such as the liver and the kidney) is phosphorylated twice, cleaved and ultimately rearranged to form two pyruvate molecules. Pyruvate can be used in several ways. One common pathway involves the continuation of cytoplasmic glycolysis with pyruvate being converted to lactate by the enzyme lactate dehydrogenase, producing two ATP molecules per molecule of pyruvate. Most often, and in the presence of oxygen however, pyruvate enters mitochondria via pyruvate dehydrogenase, is converted to acetate, linked to Coenzyme A to form acetyl CoA and combined with oxaloacetate to form citrate. The process by which citrate contributes to the process, also called the citric acid cycle, the tricarboxylic acid (TCA) cycle or the Krebs cycle (after Hans Krebs, the biochemist who, along with Albert Szent-Gyorgyi, first described the process), involves enzymes located within the mitochondrial matrix (Fig 2). During these enzymatic reactions, reducing equivalents are created and stored in the form of nicotinamide adenine dinucleotide dihydride (NADH-H+), flavin adenine dinucleotide hydride (FADH2+) or coenzyme Q (CoQ). The net energy product from the Krebs cycle is minor. However, the electron carriers (FADH2, NADHH+ and $\mathrm{CoQ}$ ) provide substrate for the electron transport chain.

The electron transport chain (Fig 3) transfers energy from carriers to form ATP. This is accomplished by passing the energy, in the form of electrons, through four protein complexes on the inner mitochondrial membrane. Coupled with electron transport, protons $(\mathrm{H}+)$ are pumped into the intra-membrane space by complexes I, III, and IV. The result is an electrochemical potential gradient, $\Delta \psi_{\mathrm{m}}$, of $180 \mathrm{mV}$. The energy from $\Delta \psi_{\mathrm{m}}$ is used by the fifth complex, ATP synthase, to convert ADP to high-energy ATP. The mobile electron carrying molecules within the chain are ubiquinone and cytochrome c. In complex IV, cytochrome $\mathrm{c}$ oxidase, electrons from cytochrome $\mathrm{c}$ are transferred to molecular oxygen. The $\mathrm{O}_{2}{ }^{-2}$ combines with two $\mathrm{H}+$ ions to form water. It is well understood that active release of cytochrome c into the cytoplasm can initiate apoptosis, or programmed cell death. However, there are data suggesting that this does not occur in sepsis within the majority of organs. In aerobic environments the amount of ATP that can be produced may be limited only by the availability of pyruvate. In order to limit the proton gradient from becoming excessive, a controlled leak back into the mitochondrial matrix is mediated by carriers called uncoupling proteins.

The most profound disruption of the proton motive fore and normal mitochondrial function occurs when the mitochondrial membrane becomes excessively permeable. This is characteristic of a number of pathological conditions and leads to the development of the socalled mitochondrial permeability transition pore (MPTP), a large, non-specific channel in 
the internal mitochondrial membrane. MPTP opening (called mitochondrial permeability transition, MPT) allows water and molecules of up to $1.5 \mathrm{kDa}$ to cross the usually impermeable internal membrane. The resulting depolarization oxidative phosphorylation, depletes ATP, promotes mitochondrial swelling and may initiate apoptosis.

Under normal conditions, mitochondria are destroyed and replaced on a regular basis. Destruction occurs by autophagy. Regeneration of mitochondria, called biogenesis, is a tightly regulated process that involves expression of a set of proteins, transfer RNAs and ribosomal RNAs that are encoded by DNA contained within the mitochondria (mtDNA). mtRNA expression, in turn, is controlled by a complex series of reactions that may involve the expression of upwards of 1000 nuclear-encoded proteins. Failed biogenesis is another source of profound disruption of mitochondrial function that is seen is pathological states, including sepsis.

\section{Mitochondrial Dysfunction in Sepsis}

\section{a. Oxygen Utilization and ATP Dynamics}

Mitochondrial impairment during critical illness has been recognized for quite some time. Hypoxia-induced mitochondrial dysfunction was first identified by Barcroft et al in 1945. This investigation identified three causes of hypoxia that led to mitochondrial dysfunction: decreased arterial oxygen tension (hypoxic hypoxia), decreased systemic hemoglobin concentration (anemic hypoxia), and/or microvascular dysfunction leading to hypoperfusion (stagnant hypoxia) [4]. However, the effects of sepsis on cellular ATP levels are unclear. Work by Hotchkiss et al in skeletal muscle suggest that sepsis depletes cellular ATP content, not from a lack of oxygen but rather because high-energy phosphate levels may be reduced [5]. In contrast, studies on muscle and liver by Brealey et al did not demonstrate ATP depletion [6,7]. Most importantly, recent investigations clearly show that sepsis impairs ATP production [8]. Therefore, the maintenance of normal levels must reflect decreased utilization.

\section{b. Microvascular Dysfunction vs. Cytopathic Hypoxia}

Initial research regarding septic pathophysiology led clinicians to propose that the key abnormality was microvascular dysfunction. This entity, involving a heterogenous alteration in perfusion, was first described by Weil et al, in 1971 [9]. The perfusion mismatch would result in failed peripheral oxygen utilization [10]. Sidestream dark-field imaging has indeed revealed an impaired microcirculation in septic patients [10]. Using this approach, Ince and colleagues found that capillary perfusion in sepsis may take any combination of five forms (Table 1). The net effect is a failure of oxygen delivery in sepsis Fink et al proposed that microvascular dysfunction may not be the abnormality that effects mitochondrial respiration during sepsis. Rather, flow may be normal or even excessive but an intrinsic derangement affecting cellular energy metabolism may preclude oxygen utilization. This defect is called cytopathic hypoxia and its existence is supported by tissue oxygen measurements and direct examination of cellular and mitochondrial respiration [6,11,12]. Mechanisms that explain cytopathic hypoxia during sepsis include impaired pyruvate delivery, inhibition of the enzymes involved in Krebs cycle and/or the electron transport chain, activation of poly(ADP)-ribosylpolymerase (PARP) and failed maintenance of the trans-mitochondrial membrane proton gradient with uncoupling of ATP synthase [6].

\section{c. Pyruvate Dehydrogenase Dysfunction}

Pyruvate dehydrogenase (PDH) E1 is catalytic component of the multimeric pyruvate dehydrogenase complex (PDC) that is responsible for synthesizing acetyl-CoA from pyruvate. PDH activity is stimulated by insulin, phosphoenolpyruvic acid and AMP and is 
inhibited by ATP, NADH and acetyl-CoA. Inactivation of the PDC will inevitably impair ATP production [13].

PDC dysfunction in sepsis was, at one time, the subject of intense investigation [14]. This reflected the demonstration of increased lactate production despite normal- to-increased skeletal muscle blood flow. Similarly, Kantrow et al, in the liver of rats made septic by cecal ligation and puncture (CLP), found decreased oxygen consumption [15] and attributed this to failed generation of substrate (glutamate, malate, succinate) for the Krebs cycle/electron transport chain [16]. Interest in PDH and the elements of the Krebs cycle has waned as focus has shifted to components of the electron transport chain. However, as will be discussed below, the abnormalities in electron transport may be adaptive. This makes it increasingly important to identify other defects.

\section{d. Altered Oxidative Phosphorylation}

Impaired ATP production could result from dysfunction in any of the four complexes of the electron transport chain. These develop a proton gradient that creates the energy potential used by ATP synthase to convert ADP into ATP $[17,11,12]$. The cycle of production and consumption continues as long as glucose is fed into the system and respiration is not inhibited.

Cytochrome c oxidase, Complex IV of the electron transport chain, consists of 13 different subunits, three of which comprise the active site where $\mathrm{O}^{-2}$ combines with $2 \mathrm{H}^{+}$molecules to form water. This action also helps generate the electrical intermembrane potential for ATP generation. The active site is characterized by a copper-containing center and two heme centers (heme a, a3) where molecular oxygen is bound. Because of its role as the final electron acceptor and the complex that uses oxygen, Complex IV is felt by many to be of prime importance, and perhaps even rate-limited. Studies in murine models of sepsis have demonstrated non-competitive inhibition of cytochrome $\mathrm{c}$ utilization. At late time points, there is a loss of heme content and a failure of subunit I expression [18]. The active subunits are encoded by mtDNA. A decrease in the abundance of these key subunits likely indicates failed biogenesis and irreversible mitochondrial dysfunction.

Experimentally, it is difficult to separate the activity Cof Complex II from that of Complex III. Therefore, most studies report on joint activity. Several investigations demonstrate sepsis-associated dysfunction of complex II/III. Brealey et al, studied electron transport activity using a rodent model of sepsis. They were unable to identify a defect in Complex II/ III activity in either liver or skeletal muscle over a period of 72 hours. However, activity of hepatic Complex I and Complex IV fell over time. In the late time point in septic animals, all complex protein activity was noted to be much lower than sham operated mice in both hepatic and muscle tissue [19].

Fredriksson et al studied mitochondrial metabolic dysfunction in septic ICU patients and compared this healthy patients undergoing elective surgery. In septic patients suffering from MODS they observed a two-fold decrease in all mitochondrial complex activity in intercostal muscle and leg muscle [20].

Inhibition of either Complex I or Complex IV can increase the production of reactive oxygen (ROS) and nitric oxide (NO) [21-25]. Production of these free radicals increases with aging as well as in individuals with immune dysfunction [26]. Both have been implicated in the pathogenesis of sepsis when excess production may overwhelm endogenous protective mechanisms [25] and may impair mitochondrial bioenergetic production $[23,24]$. ROS and NO in excess also may induce mitochondrially-mediated apoptosis. 


\section{Resuscitation}

As discussed, Complex IV is inhibited in septic mice. Piel et al, administered exogenous cytochrome c (cyt c) and examined the effect on mitochondrial dysfunction and myocardial contractility [27]. These studies revealed that cyt c given 24 hours after the induction of sepsis via CLP abrogated the inhibition of Complex IV and improved cardiac contractility. Further, 50\% of cyt c injected mice survived to 96 hours in contrast to a survival rate of only $15 \%$ following saline injection [28]. Interestingly, despite the demonstrated role of cyt $\mathrm{c}$ in apoptosis, no increase in programmed cell death was noted.

In addition to exogenous cyt $\mathrm{c}$, we have examined the effects of caffeine on sepsis-induced mitochondrial dysfunction. Verma et al demonstrated that the intraperitoneal injection of 7.5 $\mathrm{mg} / \mathrm{kg}$ of caffeine (equivalent to the concentration in an average cup of coffee) to mice following CLP increased Complex IV kinetic activity to levels equivalent to those seen following sham operation [29].

\section{Conclusion - Is Early Sepsis-Induced Mitochondrial Inhibition Adaptive?}

The above data demonstrate a clear role for mitochondrial dysfunction in the pathogenesis and pathophysiology of sepsis. What is less clear is the teleology underlying this response. Prolonged mitochondrial dysfunction and impaired biogenesis unquestionable are detrimental. However, early inhibition of mitochondrial function may serve another purpose.

Following experimental ischemia-reprefusion injury and clinically after myocardial infarction, the heart enters a state of reduced metabolism and contractility. This response is referred to as "hibernation" and is viewed to be adaptive, allowing demarcation of irreversibly effected muscle and recovery of tissue that is incompletely effected [30]. Use of hydrogen sulfide [31] and S-nitrosothiols (SNOs) [32], which inhibit Complex IV and Complex I, respectively, have been shown to induce a similar state and protect against ischemia-reperfusion injury. It is attractive to postulate that sepsis-induced reductions in organ function are similarly protective [17]. This concept, termed "metabolic quiescence" proposes that, in response to some sepsis-induced injury, the production of nitric oxide (NO), carbon monoxide (CO) or some other molecular species that binds to the heme moiety in Complex IV reversibly inhibits the function of this enzyme. This hypothesis is supported by the sepsis-induced 1) overproduction of $\mathrm{NO}$ and $\mathrm{CO}, 2$ ) decrease in ATP production [8], 3) lack of irreversible damage following recovery, 3) demonstration of non-competitive inhibition Complex IV in septic myocardium [18] and liver [unpublished data] and 4) demonstration of increased glycogen deposition, enhanced expression of the GLUT4 receptor and a switch in myocardial substrate preference from fatty acids to glucose, all characteristic of hibernation following ischemia-reperfusion injury.

The "mitochondrial inhibition as an adaptive response" hypothesis is intriguing but remains unproven. What is clear is that investigation into the role of mitochondria in the pathogenesis and pathophysiology of sepsis represents an exciting avenue of future investigation.

\section{References}

1. Angus, Derek C.; Linde-Zwirble, Walter T.; Lidicker, Jeffrey, et al. Epidemiology of severe sepsis in the United States: Analysis of incidence, outcome, and associated costs of care. Critical Care Medicine 2001;29:1303-1310. [PubMed: 11445675]

2. Members of the American college of Chest Physicians /Society of Critical Care Consensus Committee. American College of Chest Physicians/Society of Critical Care Medicine Consensus 
Conference: Definitions of sepsis and organ failure and guidelines for the use of innovative therapies in sepsis. Critical Care Medicine 1992;20:864-874. [PubMed: 1597042]

3. Levy, Mitchell M.; Fink, Mitchell P.; Marshall, John C., et al. 2001 SCCM/ESICM/ACCP/ATS/SIS International sepsis definition conference. Critical Care Medicine 2003;31:1250-1256. [PubMed: 12682500]

4. Barcroft H, Allen WJ, Anderson DP, et al. Circulatory changes during fainting and coma caused by oxygen lack. Journal of Physiology 1946;104:426-434. [PubMed: 16991699]

5. Hotchkiss, Richard S.; Karl, Irene E. Reevaluation of the role of cellular hypoxia and bioenergetic failure in sepsis. Journal of the American Medical Association 1992;267:1503-1510. [PubMed: 1538541]

6. Fink, Mitchell P. Cytopathic hypoxia: Mitochondrial dysfunction as mechanism contributing to organ dysfunction in sepsis. Critical Care Clinics 2001;17:219-237. [PubMed: 11219231]

7. Brealey, David; Karyampudi, Sekhar; Jacques, Thomas S., et al. Mitochondrial dysfunction in a long-term rodent model of sepsis and organ failure. American Journal of Physiology 2004;286:R491-R497. [PubMed: 14604843]

8. López LC, Escames G, Ortiz F, et al. Melatonin restores the mitochondrial production of ATP in septic mice. Neurology and Endocrinology Letters 2006;27:623-630.

9. Weil MH, Shubin H. Proposed reclassification of shock states with special reference to distributive effects. Advance Experiments in Medical Biology 1971;23:13-23.

10. Elbers, Paul WG.; Ince, Can. Bench-to-bedside review: Mechanisms of critical illness - classifying microcirculatory flow abnormalities in distributive shock. Critical Care Clinics 2006;10:221.

11. Fink, Mitchell P. Bench-to-bedside review: Cytopathic hypoxia. Critical Care Clinics 2002;6:491499.

12. Fink, Mitchell P. Cytopathic hypoxia. Is oxygen use impaired in sepsis as a result of acquired intrinsic derangement in cellular respiration? Critical Care Clinics 2002;18:165-175. [PubMed: 11910729]

13. Vary, Thomas C.; Siegel, John H.; Nakatani, Toshio, et al. Effect on sepsis on activity of pyruvate dehydrogenase complex in skeletal muscle and liver. American Journal of Physiology Endocrinology and Metabolism 1986;250:E634-E640.

14. Vary, Thomas C.; Hazen, Stacy. Sepsis alters pyruvate dehydrogenase kinase activity in skeletal muscle. Molecular and Cellular Biochemistry 1999;198:113-118. [PubMed: 10497885]

15. Kantrow, Stephen P.; Taylor, David E.; Carraway, Martha S., et al. Oxidative metabolism in rat hepatocytes and mitochondria during sepsis. Archives of Biochemistry and Biophysics 1997;345:278-288. [PubMed: 9308900]

16. Kantrow, Stephen P.; Tatro, Lynn G.; Piantidosi, Claude A. Oxidative stress and adenine nucleotide control of mitochondrial permeability transition. Free Radical Biology \&amp; Medicine 2000;28:251-260. [PubMed: 11281292]

17. Levy, Richard J. Mitochondrial dysfunction, bioenergetic impairment, and metabolic downregulation in sepsis. Shock 2007;28:24-28. [PubMed: 17483747]

18. Levy, Richard J.; Vijayasarathy, C.; Raj, Nichelle R., et al. Competitive and noncompetitive inhibition of myocardial cytochrome $c$ oxidase in sepsis. Shock 2004;21:110-114. [PubMed: 14752282]

19. Brealey, David; Karyampudi, Sekhar; Jacques, Thomas S., et al. Mitochondrial dysfunction in a long-term rodent model of sepsis and organ failure. American Journal of Physiology 2004;286:R491-R497. [PubMed: 14604843]

20. Fredriksson, Katarina; Hammarqvist, Folke; Strigard, Karin, et al. Derangements in mitochondrial metabolism in intercostal and leg muscle of critically ill patients with sepsis-induced multiple organ failure. American Journal of Physiology 2006;291:E1044-E1050. [PubMed: 16803854]

21. Brealey, David; Brand, Michael; Hargreaves, Lain, et al. Association between mitochondrial dysfunction and severity and outcome of septic shock. Lancet 2002;360:219-223. [PubMed: 12133657]

22. Clementi, Emilio; Brown, Guy C.; Feelisch, Martin, et al. Persistent inhibition of cell respiration by nitric oxide: Crucial role of S-nitrosylation of mitochondrial complex I and protective action of glutathione. Proceedings of the National Academy of the Sciences 1998;95:7631-7636. 
23. Bolanos, Juan P.; Heales, Simon J.; Peuchen, Stefan, et al. Nitric oxide-mediated mitochondrial damage: A potential neuroprotective role for glutathione. Free Radical Biology \&amp; Medicine 1996;21:995-1001. [PubMed: 8937884]

24. Hoffman, David L.; Brookes, Paul S. Oxygen sensitivity of mitochondrial reactive oxygen species generation depends on metabolic conditions. Journal of Biological Chemistry 2009;284:1623616245. [PubMed: 19366681]

25. Taylor, David E.; Ghio, Andrew J.; Piantadosi, Claude A. Reactive oxygen species produced by liver mitochondria of rats in sepsis. Archives of Biochemistry and Biophysics 1995;316:70-76. [PubMed: 7840680]

26. Rada, Balázs; Leto, Thomas L. Oxidative innate immune defenses by Nox/Duox family NADPH Oxidases. Contributions in Microbiology 2008;15:164-187.

27. Piel, David A.; Gruber, Peter J.; Weinheimer, Carla J., et al. Mitochondrial resuscitation with exogenous cytochrome $\mathrm{c}$ in the septic heart. Critical Care Medicine 2007;35:2120-2127. [PubMed: 17855825]

28. Piel, David A.; Deutschman, Clifford S.; Levy, Richard J. Exogenous cytochrome c restores myocardial cytochrome oxidase activity into the late phase of sepsis. Shock 2008;29:612-616. [PubMed: 18414235]

29. Verma, Richa; Huang, Zhishan; Deutschman, Clifford S., et al. Caffeine restores myocardial cytochrome oxidase activity and improves cardiac function during sepsis. Critical Care Medicine 2009;37:1397-1402. [PubMed: 19242342]

30. Zivadinovic, Dragoslava; Marjanovic, Marina; Andjus, Radoslav K. Some components of hibernation rhythms. Annals of the New York Academy of Science 2005;1048:60-68.

31. Blackstone, Eric; Morrison, Mike; Roth, Mark B. H2S induces a suspended animation-like state in mice. Science 2005;308:518. [PubMed: 15845845]

32. Burwell, Lindsay S.; Nadtochiy, Sergiy M.; Brookes, Paul S. Cardioprotection by metabolic shutdown and gradual wake- up. Journal of Molecular and Cellular Cardiology 2009;46:804-810. [PubMed: 19285082]

33. Pelicano H, Martin DS, Xu R-H, et al. Glycolysis inhibition for anticancer treatment. Oncogene 2006;25:4633-4646. [PubMed: 16892078]

34. Bender, David A.; Mayes, Peter A. Harper's Illustrated Biochemsitry 28 ed. The McGraw-Hill Companies; 2009. Chapter 18, Glycolysis and the Oxidation of Pyruvate. ISBN 978-0-07-162591-3

35. Protti, Alessandro; Singer, Mervyn. Bench-to-bedside review: Potential strategies to protect or reverse mitochondrial dysfunction in sepsis-induced organ failure. Critical Care Clinics 2006;10:228. 




1,3-bisphosphoglycerate

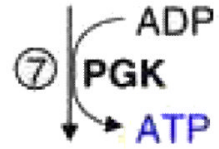

3-Phosphoglycerate

(8) $\downarrow$ PGM

2-Phosphoglycerate

(9)|Enolase

Phosphoenolpyruvate
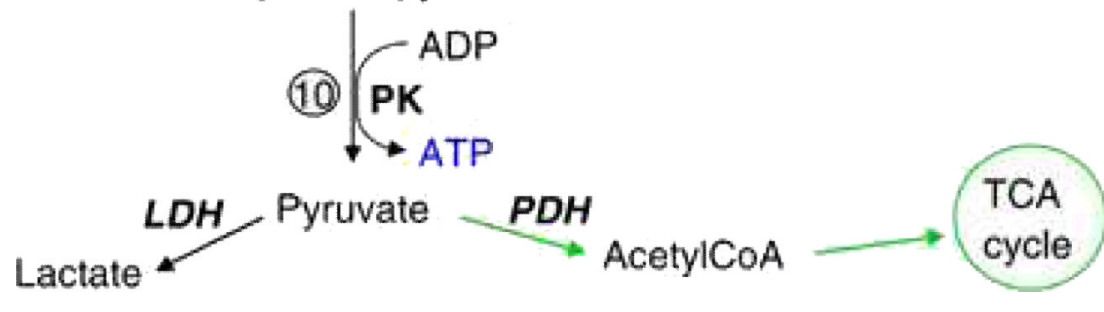

Fig. 1.

Glycolytic Pathway (30). 




Fig. 2.

Citric Acid Cycle (31). 


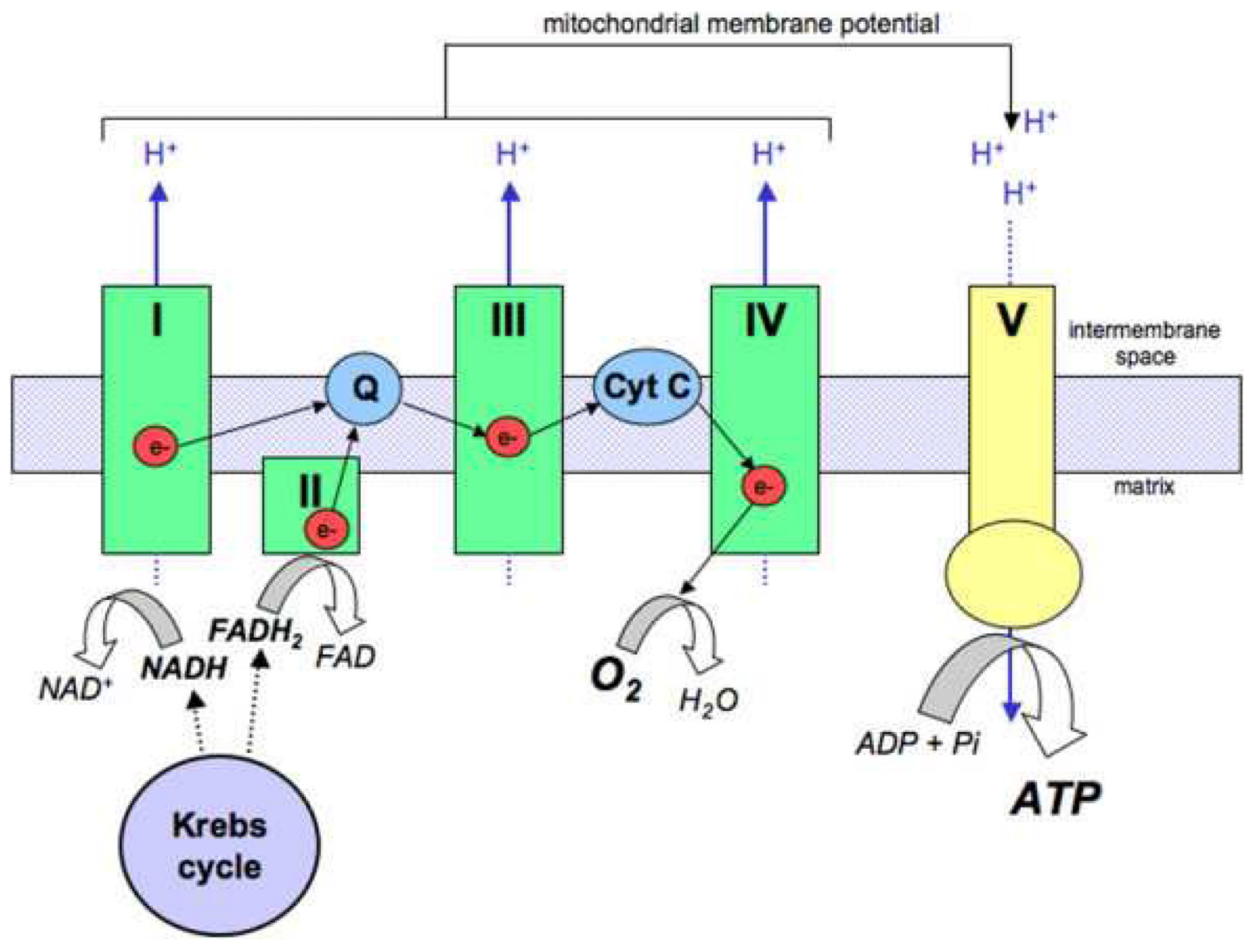

Fig. 3.

The Electron Transport Chain (32). 


\section{Table 1}

Microvascular Flow Patterns in Sepsis (after ref 10)

\begin{tabular}{ll}
\hline Stagnant & blood moving in venules but not capillaries \\
Empty/Continuous & blood moving in venules with no blood in some capillaries and free flow in others \\
Stagnant/Continuous & blood moving in venules with impaired flow in some capillaries and free flow in others \\
Stagnant/hyperdynamic & hyperdynamic flow in venules, stagnant flow in some capillaries and hyperdynamically in others \\
Hyperdynamic & hyperdynamic flow in both venules and capillaries. \\
\hline
\end{tabular}

